MSUPHY99.08

\title{
Using binary stars to bound the mass of the graviton
}

\author{
Shane L. Larsonl and William A. Hiscock \\ Department of Physics, Montana State University, Bozeman, \\ Montana 59717
}

(December 22, 1999)

\begin{abstract}
Interacting white dwarf binary star systems, including helium cataclysmic variable $(\mathrm{HeCV})$ systems, are expected to be strong sources of gravitational radiation, and should be detectable by proposed space-based laser interferometer gravitational wave observatories such as LISA. Several HeCV star systems are presently known and can be studied optically, which will allow electromagnetic and gravitational wave observations to be correlated. Comparisons of the phases of a gravitational wave signal and the orbital light curve from an interacting binary white dwarf star system can be used to bound the mass of the graviton. Observations of typical HeCV systems by LISA could potentially yield an upper bound on the inverse mass of the graviton as strong as $h / m_{g}=\lambda_{g}>1 \times 10^{15} \mathrm{~km}\left(m_{g}<1 \times 10^{-24} \mathrm{eV}\right)$, more than two orders of magnitude better than present solar system derived bounds.
\end{abstract}

Typeset using REVTEX 


\section{INTRODUCTION}

The advent of large scale laser interferometer gravitational wave detectors promises to lead to the direct detection of gravitational radiation, which will create a new observational field of science: gravitational wave astronomy. The introduction of gravitational waves into the retinue of astronomical observations will yield important new information about the dynamics of astrophysical systems, and will also provide an excellent opportunity to conduct new tests of gravity.

Proposed space-based laser interferometers, such as the LISA (Laser Interferometer Space Antenna) [1] and OMEGA (Orbiting Medium Explorer for Gravitational Astrophysics) [2] observatories, will be particularly well poised to begin astrophysical studies since there are known sources of gravitational radiation which will be easily visible to these instruments, namely interacting binary white dwarf (IBWD) star systems.

The IBWD sources are particularly appealing targets because they are good candidates for simultaneous optical and gravitational wave observations. Many of the IBWDs which are known to exist are being studied and monitored by the Center for Backyard Astrophysics (CBA)円, and are expected to be strong sources of monochromatic gravitational waves which should be easily visible to an instrument such as LISA with only a few minutes of signal integration. Simultaneous optical and gravitational wave observations will be useful in refining the current physical models used to describe these systems, and for testing relativistic theories of gravity in the radiative regime by comparing the propagation speeds of electromagnetic and gravitational wave signals.

This paper examines how the comparison of the phase of the orbitally modulated electromagnetic signal (the light curve) and a gravitational wave signal from an IBWD star system

\footnotetext{
${ }^{1}$ The CBA is a network of amateur astronomers equipped with CCD photometry equipment who monitor variable stars. The network is managed by professional astronomers at Columbia University.
} 
can be used to bound the mass of the graviton. If the mass of the graviton is assumed to be known by other measurements, then the observations may be used to determine the properties of the binary star system being monitored.

Current conservative bounds on the graviton mass come from looking for violations of Newtonian gravity in surveys of planetary motions in the solar system. If gravity were described by a massive field, the Newtonian potential would have Yukawa modifications of the form

$$
V(r)=-\frac{M}{r} \exp \left(-r / \lambda_{g}\right)
$$

where $M$ is the mass of the source of the potential, and $\lambda_{g}=h / m_{g}$ is the Compton wavelength of the graviton, where $m_{g}$ is the graviton mass. The current best bound on the graviton mass from planetary motion surveys is obtained by using Kepler's third law to compare the orbits of Earth and Mars, yielding $\lambda_{g}>2.8 \times 10^{12} \mathrm{~km}\left(m_{g}<4.4 \times 10^{-22} \mathrm{eV}\right)$ [3.

Another bound on the graviton mass can be established by considering the motions of galaxies in bound clusters [4], yielding $\lambda_{g}>6 \times 10^{19} \mathrm{~km}\left(m_{g}<2 \times 10^{-29} \mathrm{eV}\right)$. This bound, while stronger than solar system estimates, is considerably less robust, due to uncertainty about the matter content of the Universe on large scales (e.g., the amount and nature of dark matter is widely debated, and uncertain at best).

Recent work by Will [5] has suggested that the mass of the graviton could be bounded using gravitational wave observations. If the graviton is a massive particle, then the speed of propagation of a gravitational wave will depend on its frequency. As binary systems evolve, they will slowly spiral together due to the emission of gravitational radiation. Over the course of time, the frequency of the binary orbit rises, ramping up rapidly in the late stages of the evolution, just prior to coalescence. Laser interferometer gravitational wave detectors should be able to track the binary system's evolution, obtaining the detailed time-dependent waveform using the matched filtering techniques required for data analysis in these detectors.

Space-based detectors such as LISA will be able to observe the coalescence of massive $(\sim$ 
$10^{5}$ to $\sim 10^{7} \mathrm{M}_{\odot}$ ) binary black holes, as well as the gravitational wave emission from compact binary star systems which are far from coalescence (e.g., interacting binary white dwarfs). Ground-based detectors such as LIGO will be able to detect the merger of smaller black hole binaries $\left(\sim 10 \mathrm{M}_{\odot}\right)$, as well as the coalescence of compact binary stars (e.g., neutron star/neutron star binaries). If the graviton is a massive particle, then the observed signal will not perfectly match theoretical templates computed using general relativity theory, in which the graviton is massless; a massive graviton would cause dispersion in the gravitational waves. By using matched filtering of inspiral waveforms, this dispersion could be bounded, thereby bounding the mass of the graviton. Will [5] finds that LIGO could bound the graviton mass at $\lambda_{g}>6.0 \times 10^{12} \mathrm{~km}\left(m_{g}<2.1 \times 10^{-22} \mathrm{eV}\right)$ by observation of the inspiral of two $10 \mathrm{M}_{\odot}$ black holes. A space-based interferometer such as LISA, observing the inspiral of two $10^{7} \mathrm{M}_{\odot}$ black holes could bound the graviton mass at $\lambda_{g}>6.9 \times 10^{16} \mathrm{~km}\left(m_{g}<1.8 \times 10^{-26} \mathrm{eV}\right)$. If the graviton is massive, then these numbers represent the minimum masses detectable by such observations.

The analysis in this paper shows that LISA observations of known IBWD sources could yield a bound as strong as $\lambda_{g}>5 \times 10^{15} \mathrm{~km}\left(m_{g}<2 \times 10^{-25} \mathrm{eV}\right)$, considerably stronger than present solar system based bounds. The IBWD bound also has the advantage of not depending on the complicated details of black hole coalescences.

Section [1] reviews what is known about the interacting binary white dwarfs, in particular the helium cataclysmic variable (HeCV) systems and their archetype, the binary AM CVn (AM Canum Venaticorum). Section [1T reviews the basic notions associated with (possibly) massive photons and gravitons. Sections $\mathbb{I V}-\mathrm{V}$ propose a new experiment to measure the graviton mass using IBWD observations, and an expression for the mass is derived. In Section VI, the sensitivity predicted for LISA is used to estimate how precise a bound could be placed on the graviton mass from HeCV observations. Section VII summarizes the results, and also suggests how correlation of phase measurements might be used to measure other astrophysical parameters in the binary system, such as the accretion disk radius.

Throughout this paper, geometric units with $G=c=1$ are employed, unless otherwise 
noted.

\section{INTERACTING BINARY WHITE DWARF SYSTEMS}

Estimates suggest the Galaxy is populated by $\sim 10^{7}$ close binary star systems [6]. The sheer numbers of these systems is likely to have profound consequences for space-based gravitational wave observatories. The combined gravitational waves from these binaries will produce a stochastic background which rises well above the low frequency detection sensitivity of LISA [7]. Particularly strong (e.g., nearby) binary systems will rise above this background and be observable by a spaceborne observatory. One class of such sources are the helium cataclysmic variable $(\mathrm{HeCV})$ stars. The properties of the six nearest known HeCVs are shown in Table [1 [8]. The predicted stochastic gravitational wave background due to short period binary stars, as calculated by Hils and Bender [7] and the predicted signals of the six nearest HeCVs are plotted in Figure 1, along with the predicted sensitivity curve of LISA. If present models of the spatial density of close binaries in the Galaxy are correct, roughly 5000 of these sources should be individually detectable by a space-based laser interferometer such as LISA [1].

Currently the best models for HeCVs describe a star system where the secondary star (the lower mass companion in the binary system, usually a degenerate helium dwarf star) has expanded to fill its Roche lobe, and the primary star (the larger mass, compact white dwarf) lies at the core of an accretion disk. Matter overflows from the secondary Roche lobe and streams onto the accretion disk, creating a hot spot which emits a strong electromagnetic signal.

There are several mechanisms whereby the light curve of an HeCV could be modulated as seen from the perspective of observers on Earth. The simplest model is for systems whose orbital plane is close to the line of sight to the Earth, so that the stars periodically eclipse, partially or wholly. The eclipse phase could dim the stellar components, part of the primary emission regions of the accretion disk, or the Roche lobe. Another possible mechanism for 
variation in the light curve is associated with the emission from the secondary star, which has expanded to fill its Roche lobe. The star will appear brighter when the largest surface area is presented to the observer along the projected line of sight. This will occur twice in each orbit, when the line of sight is perpendicular to the line of centers. Signatures in the light curve due to projected area effects are called "ellipsoidal variations," and are most easily observed in infrared wavelengths [9]. Yet another possible mechanism is currently favored to explain the source of variation in the light curve of the archetype system for HeCVs, AM CVn [10]. In this model the hot spot on the accretion disk radiates approximately radially outward from the disk. As the binary orbits, this hot spot alternately turns towards and away from distant observers, leading to a modulation of the light curve (a so-called "flashlight" mechanism).

A detailed theoretical model of AM CVn has been constructed, describing a variety of signals which are present in the photometric data 11 13. This model suggests that AM $\mathrm{CVn}$ is a member of a class of variable stars that have periodic features in the light curve known as "superhumps" [14,15]. The model explains the superhump feature as being caused by the existence of an eccentric precessing accretion disk, with a precession period which is slightly longer than the orbital period of the binary. Knowing the superhump period, $P_{s h}$, and the precessional period of the accretion disk (apsidal advance), $P_{a a}$, the model predicts the orbital period will be given by

$$
P_{o r b}^{-1}=P_{s h}^{-1}+P_{a a}^{-1}
$$

Photometry of AM CVn shows the existence of a superhump signature at $P_{s h}=1051.2 \mathrm{~s}$, and the period of the accretion disk precession at $P_{a a}=13.38 \mathrm{hr}$. Using Eq. (2) this model predicts a binary orbital period of $P_{\text {orb }}=1028.77 \pm 0.18 \mathrm{~s}$ for AM CVn [13]. Photometric observations by the CBA have recently confirmed an orbital period of $P_{\text {orb }}=1028.7325 \pm$ $0.0004 \mathrm{~s}$ [10]. 


\section{MASSIVE GRAVITONS AND PHOTONS}

To constrain the mass of the graviton by comparing the propagation speed of gravitational and electromagnetic waves, one must consider how the speed of gravitational waves (and electromagnetic waves) is related to the mass of the graviton (and the possible mass of the photon).

The current bound on the photon mass is $m_{\gamma}<2 \times 10^{-16} \mathrm{eV}$ [16], which is much larger than the current bounds on the graviton mass $(c f$. , the solar system bound on the graviton mass is $\left.m_{g}<4.4 \times 10^{-22} \mathrm{eV}\right)$. Is it then justifiable to treat the photon as a massless particle, while at the same time treating the graviton as a massive particle?

The resolution to this question can be understood by examining the partition of energy between the rest mass and kinetic energy of a particle being received from a distant binary. From the relativistic energy, $E^{2}=p^{2}+m^{2}$, one may write the velocity of any particle as

$$
v^{2}=1-\frac{m^{2}}{E^{2}}
$$

If $m \ll E$, then Eq. (3) implies

$$
\varepsilon=1-v \simeq \frac{1}{2} \frac{m^{2}}{E^{2}}
$$

where $\varepsilon$ parameterizes the difference between the velocity of the particle and $c$.

For optical photons $(\lambda \sim 500 \mathrm{~nm})$ received from a binary star system, the characteristic energy is $E_{\gamma} \simeq 2.5 \mathrm{eV}$. For this energy, $\varepsilon_{\gamma} \leq 3 \times 10^{-33}$. Similar considerations may be applied to the gravitons received from the same binary systems. In this case, the frequency

of the gravitational waves is $f \sim 10^{-3} \mathrm{~Hz}$, giving a characteristic energy for a single graviton of $E_{g} \sim 4 \times 10^{-18} \mathrm{eV}$. Using the solar system bound on the graviton mass, $m_{g}<4.4 \times 10^{-22}$ $\mathrm{eV}$, yields $\varepsilon_{g} \leq 1 \times 10^{-8}$.

For the current bounds on the photon and graviton masses, $\varepsilon_{\gamma} \ll \varepsilon_{g}$. If the bound on the mass of the graviton is not drastically improved (e.g., decreasing the bound on $m_{g}$ by 12 orders of magnitude, such that $\varepsilon_{g} \sim \varepsilon_{\gamma}$ ), then the effect of a non-zero mass will be much 
more significant for gravitons than photons in our analysis. This justifies the treatment of the photons as massless particles and the gravitons as massive particles in this paper.

Since the gravitational waves emitted by an HeCV binary star system are essentially monochromatic, they will have a single particular velocity,

$$
v_{g} \simeq 1-\frac{1}{2}\left(\frac{m_{g}}{E}\right)^{2}
$$

Writing the energy in Eq. (5) as $E=\hbar \omega$, and identifying the Compton wavelength of the graviton as $\lambda_{g}=h / m_{g}$ this becomes

$$
v_{g} \simeq 1-\frac{1}{2}\left(\frac{2 \pi}{\lambda_{g} \omega}\right)^{2} .
$$

\section{CORRELATION OF ELECTROMAGNETIC AND GRAVITATIONAL OBSERVATIONS TO MEASURE THE GRAVITON MASS}

As noted in Section [1, it is expected that the Galaxy harbors a large population of interacting binary white dwarf stars. The light curves for several of these systems are already known, obtained from ground-based optical photometry. Because these systems are expected to be observable in the gravitational wave spectrum (in addition to the optical spectrum), they present an excellent opportunity to directly compare the propagation speed of electromagnetic and gravitational waves.

Consider the schematic diagram shown in Figure 2. The phase fronts of the light curve modulation are represented in the top half of the diagram. The binary star system will also emit gravitational radiation which could be monitored by Earth-bound observers as well. The phase fronts of the gravitational wave signal are represented in the lower half of the diagram?.

\footnotetext{
${ }^{2}$ Assuming a circular orbit, the frequency of the gravitational radiation is actually twice the orbital frequency of the binary. For clarity, only half the gravitational phase fronts have been drawn in the diagram.
} 
Suppose the two signals are emitted in phase at the source as shown. If the graviton is a massive particle, then the gravitational waves propagate at a speed $v_{g}<1$, and the gravitational phase fronts will lag behind the light curve phase fronts when the signals arrive at Earth, as shown. By measuring the lag between the phase fronts, the mass of the graviton can be measured or bounded.

To determine the lag between the two signals, the phase of each signal must be measured. Consider a binary with orbital frequency $\omega_{o}$ at a distance $D$ from Earth. Assuming the photon to be massless $(v=c=1)$, the observed phase of the light curve will be

$$
\phi_{e m}=\omega_{o} D+A,
$$

where the term $A$ represents a variety of effects (discussed in Section $\square$ ) which could create phase delays between the electromagnetic and gravitational signals that are being monitored. In contrast, the gravitational wave signal, traveling at $v_{g}<1$ will arrive at Earth with a phase of

$$
\phi_{g w}=2 \omega_{o} \frac{D}{v_{g}} .
$$

The phase lag, $\Delta$, between the light curve and gravitational wave signals is constructed from these two phases:

$$
\begin{aligned}
\Delta & =\frac{1}{2} \phi_{g w}-\phi_{e m} \\
& =\omega_{o} D\left(\frac{1}{v_{g}}-1+\alpha\right),
\end{aligned}
$$

where $\alpha=A /\left(\omega_{0} D\right)$. The factor of $1 / 2$ insures that the phase subtraction is done between two signals with the same frequency. It is convenient to define the fractional change in the phase as

$$
\epsilon=\frac{\Delta}{\omega_{o} D}=\left(\frac{1}{v_{g}}-1+\alpha\right) .
$$

Taking the definition of $v_{g}$ from Eq. (6) and substituting into Eq. (10), the Compton wavelength of the graviton as a function of the fractional phase lag is found to be 


$$
\lambda_{g}=\frac{\pi}{\omega_{o}} \sqrt{\frac{1}{2}\left(1+\frac{1}{\epsilon-\alpha}\right)} .
$$

An obvious question to ask about this analysis is how to determine whether or not the phase difference between the two signals is greater than a single cycle, and hence undetectably large $(e . g ., \Delta=2 n \pi+\xi$, where $\xi$ is a small quantity). One can eliminate this concern because strong bounds on the graviton mass already exist. The largest observable phase shift which is consistent with current bounds can be computed by simply evaluating

Eq. (11) with $\lambda_{g}$ equal to the bound of interest. For example, the bound on the graviton mass given by solar system constraints, applied to the AM CVn system, yields a maximum fractional phase change of $\epsilon=1.5 \times 10^{-9}$. Since AM CVn lies at a distance of $D=101 \mathrm{pc}$, this value of $\epsilon$ indicates a maximum phase difference of $\Delta=9.6 \times 10^{-2}$ for that system (we have let $\alpha \rightarrow 0$ here for convenience; if the measured phase difference were larger than this value, it would indicate that $\alpha \neq 0)$.

\section{PHASE DELAYS}

In order to evaluate Eq. (11), one must not only measure the phase lag between the two signals, but an estimate must be made for the value of $\alpha$.

The parameter $\alpha$ can be written as the sum of two primary sources of delay between the gravitational and electromagnetic signal phases:

$$
\alpha=\alpha_{\star}+\alpha_{\text {path }}
$$

where $\alpha_{\text {path }}$ is a phase lag associated with the wave's propagation from the binary to the observer at the Earth and $\alpha_{\star}$ is a phase lag which depends on the specific astrophysical nature of the binary star system.

In principle, $\alpha_{\text {path }}$ will be nonzero because the line of sight to the binary is an imperfect vacuum, with non-unit index of refraction. The variations in index of refraction over the path will cause a lag in the electromagnetic signal. The dominant source of this lag will be caused by propagation of the signal through the Earth's atmosphere. 
A simple estimate of the value of $\alpha_{\text {path }}$ can be made by computing the electromagnetic phase delay due to propagation through a modeled exponential atmosphere, with a density profile

$$
\rho(r)=\rho_{o} \exp \left(-r / h_{s}\right)
$$

where $h_{s}$ is the scale height of the atmosphere. If the index of refraction, $n$, is assumed to vary linearly with the density, then

$$
n(r)=1+\eta \exp \left(-r / h_{s}\right)
$$

where $\eta=\left(n_{\text {atm }}-n_{v a c}\right)=\left(n_{a t m}-1\right)$ is the difference in index of refraction between the atmosphere and vacuum. The index of refraction is related to the signal's propagation speed $v$ by

$$
v=\frac{d r}{d t}=\frac{1}{n(r)}
$$

Eq. (15) can be integrated using Eq. (14) to obtain

$$
\int_{0}^{t_{\text {transit }}} d t=\int_{0}^{r_{o}} d r\left[1+\eta \exp \left(-r / h_{s}\right)\right]
$$

where $t_{\text {transit }}$ is the time it takes a photon to transit the atmosphere, and $r_{o}$ is the height at which the effects of the atmosphere become negligible. Completing the integration yields

$$
t_{\text {transit }}=r_{o}+h_{s} \eta\left(1-e^{-r_{o} / h_{s}}\right)
$$

In order to compute a phase delay, one is interested in the time by which the photons are delayed by the atmosphere, which is

$$
\begin{aligned}
t_{\text {delay }} & =t_{\text {transit }}-t_{\text {vacuum }} \\
& =t_{\text {transit }}-r_{o}
\end{aligned}
$$

where $t_{\text {vacuum }}$ is the time it would take a photon to travel the same distance (i.e., the atmospheric depth) in vacuum. 
The phase delay introduced by this effect is obtained by multiplying the delay time by the frequency of the signal being observed,

$$
\phi_{\text {delay }}=\omega_{o} t_{\text {delay }} .
$$

The parameter $\alpha_{\text {path }}$ is obtained from the phase delay by dividing by the reference phase, $\omega_{o} D$ (as in Eq. (9)), giving

$$
\alpha_{\text {path }}=\frac{\phi_{\text {delay }}}{\omega_{o} D}=\frac{t_{\text {delay }}}{D} .
$$

It is now possible to numerically estimate the delay introduced by the atmosphere. Assuming the Earth's atmosphere to be in hydrostatic equilibrium gives a scale height $h_{s}=8500 \mathrm{~m}$. A typical value for the difference between the atmosphere's index of refraction and unity, evaluated at sea level, is $2.8 \times 10^{-4}$. Taking the atmospheric depth to be $r_{o}=10 h_{s}$, Eq. (18) gives $t_{\text {delay }}=8.0 \times 10^{-9} \mathrm{~s}$. For the prototypical source AM CVn, at a distance $D=101 \mathrm{pc}$, Eq. (20) yields $\alpha_{\text {path }}=7.7 \times 10^{-19}$. This is much smaller than any possible phase measurement, either electromagnetic or gravitational, and so we will henceforth ignore this source of uncertainty.

The parameter $\alpha_{\star}$ is a measure of the initial phase difference at the source between the gravitational wave and electromagnetic signals. It indicates the relative phase difference between the peaks in the light curve of the binary, and the peaks in the quadrupole gravitational radiation pattern. Determining the value of $\alpha_{\star}$ requires knowledge of the position of the stars in the binary system when the electromagnetic signal peaks. The quadrupole gravitational radiation pattern will peak along the line of masses in the binary system, and also $180^{\circ}$ away from the line of masses (since the frequency of the gravitational radiation is $\omega=2 \omega_{o}$ ). If the primary variation in the binary light curve is associated with the transit of the line of masses across the observer's line of sight (e.g., the system is an eclipsing binary), then it is straightforward to assign $\alpha_{\star}=0$, indicating no initial phase delay between the gravitational wave signal and the binary's light curve.

For more complicated systems such as $\mathrm{AM} \mathrm{CVn}$, the light curve variation reflects the orbital motion of the hot spot on the edge of the accretion disk where the matter stream 
from the secondary Roche lobe overflow strikes the disk. Studies of Roche lobe overflow [17] show that the transferred material remains in a coherent matter stream which spirals in towards the primary star. In this sort of system, the location of the hot spot will determine the value of $\alpha_{\star}$, which will describe the amount by which the hot spot leads the line of masses, as shown in Figure 3 .

Estimates of the size of the primary accretion disk in HeCV type systems suggest that disk radii will be around $75 \%$ of the primary Roche radius [18], but this estimate is only certain to within about $10 \%$. This uncertainty makes it virtually impossible to estimate $\alpha_{\star}$ adequately for use in Eq. (11) from present observational data. Future observations of HeCV systems, either from advanced ground-based instruments such as the Keck Interferometer, or from space-based instruments such as the Space Interferometry Mission [19] and the Terrestrial Planet Finder [20] could allow direct measurement of $\alpha_{\star}$ by optically imaging the detailed structure of close binary systems.

For cases when $\alpha_{\star}$ cannot be accurately determined, the dependence of the Compton wavelength of the graviton on this parameter (or on $\alpha_{\text {path }}$ ) can be eliminated by subtraction of two observations of the source. Consider the situation shown in Figure 1 , where the gravitational and electromagnetic signals are monitored when the Earth lies on one side of its orbit, and again six months later, when it lies on the other side of its orbit.

When the Earth is in position 1, the phase difference between the electromagnetic and gravitational wave signal can be written

$$
\Delta_{1}=\frac{\omega_{o} D}{v_{g}}-\omega_{o} D+A .
$$

Similarly, when the Earth is in position 2, the phase difference may be written

$$
\Delta_{2}=\frac{\omega_{o}(D+L)}{v_{g}}-\omega_{o}(D+L)+A,
$$

where $L \leq 2 \mathrm{AU}$ is the path length difference for the time of flight between the two measurement. Subtraction eliminates the unknown quantity $A$, yielding

$$
\Delta_{2}-\Delta_{1}=\omega_{o} L\left(\frac{1}{v_{g}}-1\right) .
$$


Defining the fractional change in phase from this quantity gives

$$
\epsilon=\frac{\Delta_{2}-\Delta_{1}}{\omega_{o} L}=\frac{1}{v_{g}}-1
$$

which in terms of the Compton wavelength becomes

$$
\lambda_{g}=\frac{\pi}{\omega_{o}} \sqrt{\frac{1}{2}\left(1+\frac{1}{\epsilon}\right)} .
$$

The unknown parameter, $\alpha$, has been eliminated from the expression for $\lambda_{g}$, but at the cost using a much shorter characteristic distance, $L \ll D$. This approach amounts to measuring the phase lag between the two signals over the time it takes to cross the Earth's orbit (at most) 3 , as opposed to the time it takes to propagate over the Earth-source distance, leading to a great loss of precision in the measurement of $\lambda_{g}$.

\section{OBTAINING A MASS BOUND}

In order to estimate a bound on the graviton mass, assume a null result for the measurement of the phase difference, $\Delta$, between the two signals. The size of $\Delta$ (and therefore $\epsilon$ ) will then be limited only by the uncertainty in the measurements of the phase. Combining the uncertainty of the gravitational phase measurements with the electromagnetic phase measurements in quadrature yields

$$
\Delta \rightarrow \delta \Delta=\sqrt{\delta \phi_{e m}^{2}+\frac{1}{4} \delta \phi_{g w}^{2}},
$$

where $\delta \phi_{e m}$ and $\delta \phi_{g w}$ are the uncertainties in each of the phases.

For observations with a space-based interferometer, the error in phase measurements can be estimated as the ratio between the sampling time and the total integration time. For

\footnotetext{
${ }^{3}$ An improvement of roughly a factor of two may be obtained by combining the Earth's orbital motion with the proper motion of the binary system relative to the Sun, which will typically be of order a few AU per year
} 
LISA, the sampling time is expected to be of order $1 \mathrm{~s}$, with total integration times of $1 \mathrm{yr}$ $=3 \times 10^{7} \mathrm{~s}$, yielding $\delta \phi_{g w}=3 \times 10^{-8}$. The CBA reports a $0.0004 \mathrm{~s}$ uncertainty over the 1028.7325s period of AM CVn, yielding a phase uncertainty of $\delta \phi_{e m}=4 \times 10^{-7}$.

For the case of AM CVn, the value of $\alpha_{\star}$ is still not known, so bounds on the graviton mass must be derived from Eq. (25). As shown in Figure 6 , the characteristic distance $L$ is simply the path length difference between the two measurements. If the inclination of the binary system to the plane of the Earth's orbit is $\beta$, then the characteristic distance is $L=2 \cos (\beta) \mathrm{AU}$. For AM CVn, which lies at ecliptic latitude $\beta=37.4^{\circ}$, this yields $L=2.38 \times 10^{11} \mathrm{~m}$. Using this value, Eq. (25) gives a bound

$$
\lambda_{g}>5 \times 10^{14} \mathrm{~m}=5 \times 10^{11} \mathrm{~km},
$$

or, in terms of the graviton mass, $m_{g}<2 \times 10^{-21} \mathrm{eV}$, about a factor of five worse than the present bound based on the motion of Mars. Even this weak bound would be of interest, however, since it is based on the dynamics of the gravitational field, (i.e., gravitational waves, rather than the static Yukawa modifications of the Newtonian potential).

If the value of $\alpha_{\star}$ could be determined precisely (e.g., by monitoring ellipsoidal variations the light curve in the infrared, as suggested in Section \1], or with future optical interferometer observations), such that the uncertainties $\delta \alpha_{\star} \lesssim 10^{-7}$, then Eq. (11) could be used to bound the graviton mass. The distance to the known IBWD systems is typically of order $D \simeq 100$ pc; combining this with a typical orbital period of $P \simeq 1500$ s yields a bound

$$
\lambda_{g}>1 \times 10^{18} \mathrm{~m}=1 \times 10^{15} \mathrm{~km},
$$

or $m_{g}<1 \times 10^{-24} \mathrm{eV}$. This potential bound would be a factor of four hundred more stringent that the present solar system based bound, and would be better than the bounds obtained from inspiraling black holes proposed by Will [5] for all but very large black holes.

During the next decade, as we await the launch of LISA, the optical astronomers may well succeed in further reducing the uncertainty in their phase measurements. If the optical signal phase error is reduced in Eq. (26) to the point where the dominant source of error is the 
gravitational wave phase measurement, then the bound obtained above could be improved by about another factor of five, to $\lambda_{g}>5 \times 10^{15} \mathrm{~km}\left(m_{g}<2 \times 10^{-25} \mathrm{eV}\right)$.

\section{SUMMARY}

After the initial detection of gravitational waves the challenge will be for the field to evolve into a productive observational science which makes firm contact with astrophysics, complementing the broad base of electromagnetic observations already supporting that field. The experiment proposed here is particularly appealing because it entails observations of known sources by space-based detectors. The existence of IBWDs has been verified (as opposed to more speculative sources, such as binary black hole coalescence events), and such objects are currently under study by observational astronomers. Detailed gravitational wave observations can begin almost as soon as a space-based interferometer such as LISA is online.

We have shown that reliable bounds on the mass of the graviton of order $\lambda_{g}>1 \times 10^{15}$ $\mathrm{km}$ could be obtained through detailed observations of the interacting binary white dwarf star systems such as AM CVn. With the combination of detailed studies of such binary systems by optical interferometers and gravitational wave observations, this could be a very robust bound, several orders of magnitude greater than the current best bounds from solar system observations.

If one assumes the graviton to be a massless particle, as predicted by general relativity, then the same measurements described here can be employed to determine the structure of the binary star system. If the graviton is massless, then any phase difference measured between the gravitational wave and electromagnetic signal must be due to effects in the binary system. Setting $v_{g}=1$ in Eq. (10) yields

$$
\epsilon=\frac{\Delta}{\omega_{o} D}=\alpha
$$

showing that the difference in phase is simply an indicator of the value of $\alpha=\alpha_{\text {path }}+\alpha_{\star}$. As

was shown in Section $V \mathbb{1}$, the value of $\alpha_{\text {path }}$ is expected to be negligible $\left(\alpha_{\text {path }}=7.9 \times 10^{-19}\right.$ for 
AM CVn). In the cases where $\alpha_{\text {path }}$ can be ignored, the measured phase difference will be a direct measure of the value of $A$, which is the amount of phase by which the electromagnetic signal leads the line of masses in the binary system. With good models of the matter stream from the secondary Roche lobe overflow (such as the trajectories shown in Figure 31), a measurement such as this could allow an accurate determination of the accretion disk radius and the refinement of physical models for HeCV type stars solely from gravitational wave observations.

\section{ACKNOWLEDGMENTS}

This work was supported in part by National Science Foundation Grant No. PHY9734834 and NASA Cooperative Agreement No. NCC5-410. 


\section{REFERENCES}

$\dagger \quad$ electronic mail address: shane@physics.montana.edu.

$\ddagger \quad$ electronic mail address: hiscock@physics.montana.edu.

[1] P. Bender et al., LISA Pre-Phase A Report (Second Edition)(1998)(unpublished).

[2] R. W. Hellings et al., Orbiting Medium Explorer for Gravitational Astrophysics (OMEGA), proposal to NASA Medium Explorer program, (1998) (unpublished).

[3] C. Talmadge et al., Phys. Rev. Lett. 61, 1159 (1988).

[4] A. S. Goldhaber and M. M. Nieto, Phys. Rev. D 9, 1119 (1974).

[5] C. M. Will, Phys. Rev. D 57, 2061 (1998).

[6] D. Hils, P. L. Bender and R. F. Webbink, Astrophys. J. 360, 75 (1990).

[7] P. L. Bender and D. Hils, Class. Quantum Grav. 14, 1439 (1997).

[8] R. W. Hellings, private communication.

[9] R. A. Wade and M. J. Ward, in Interacting binary stars, eds. J. E. Pringle and R. A. Wade, 129 (Cambridge, Cambridge, 1985).

[10] D. Harvey et al., Astrophys. J. 493, L105 (1998).

[11] J. Faulkner, B. Flannery and B. Warner, Astrophys. J. 175, L79 (1972).

[12] J. Patterson, J. Halpern and J. Raymond, Astrophys. J. 384, 234 (1992).

[13] J. Patterson, J. Halpern and A. Shambrook, Astrophys. J. 419, 803 (1993).

[14] N. Vogt, Astrophys. J. 252, 653 (1982).

[15] R. Whitehurst, Mon. Not. R. Astr. Soc. 232, 35 (1988).

[16] C. Caso et al., Euro. Phys. J. C3, 1 (1998). 
[17] B. Flannery, Mon. Not. R. Astr. Soc. 170, 325 (1975).

[18] M. E. Sulkanen, L. W. Brasure and J. Patterson, Astrophys. J. 244, 579 (1981).

[19] The Space Interferometry Mission, Final report of the Space Interferometry Science Working Group, Deane Peterson, Chair (1996).

[20] C. Beichman, NASA's TPF: The Search for (Habitable) Planets, submitted to the 193rd meeting of the American Astronomical Society (1999). 


\section{TABLES}

TABLE I. Properties of the six nearest interacting binary white dwarfs.

\begin{tabular}{lcccc}
\hline \hline & $\begin{array}{c}\text { Secondary Mass } \\
\text { Name }\end{array}$ & Orbital Period & Distance & Strain $h$ \\
\hline & & $\left(M_{\odot}\right)$ & $(\mathrm{sc})$ & $\left(\times 10^{-22}\right)$ \\
AM CVn & 0.044 & & & \\
EC15330-1403 & $\sim 0.04$ & 1028.73 & 101 & 5.27 \\
CR Boo & $\sim 0.03$ & 1119 & 165 & 5.36 \\
V803 Cen & $\sim 0.03$ & 1491 & 157 & 2.82 \\
CP Eri & $\sim 0.03$ & 1611 & $\sim 100$ & 4.20 \\
GP Com & $\sim 0.02$ & 1724 & 165 & 4.02 \\
\hline \hline
\end{tabular}




\section{FIGURES}

FIG. 1. The background of close binaries in the Galaxy, plotted with the projected sensitivity of LISA. The six most well understood AM CVn type binaries are indicated. The assumed bandwidth is $10^{-7} \mathrm{~Hz}$.

FIG. 2. Schematic of a binary star system which has observable electromagnetic and gravitational wave signals. If the graviton is a massive particle, then the phase fronts of the gravitational signal will lag behind those of the electromagnetic signal.

FIG. 3. The Roche lobe of the primary star is shown as a dark line; matter overflows from the secondary star in a coherent matter stream, shown as trajectories falling towards the white dwarf (indicated by $\star$ at the center of the Roche lobe; figure adapted from Flannery(1975) ). Depending on the radius of the accretion disk, the matter stream will strike the disk at some angle $\theta$ which leads the binary axis; this angle is directly proportional to the phase delay parameter $\alpha_{\star}$. The dashed circle represents an accretion disk radius which is $70 \%$ of the Roche radius. A $10 \%$ uncertainty in the radius of the accretin disk will yield $\sim 5^{\circ}$ uncertainty in the value of $\theta$.

FIG. 4. Schematic showing two observations of a source from opposite sides of the Earth's

orbit. The radius of the Earth's orbit is $r_{E}$ and the path length difference for signals propagating from a distant source is $L$. 


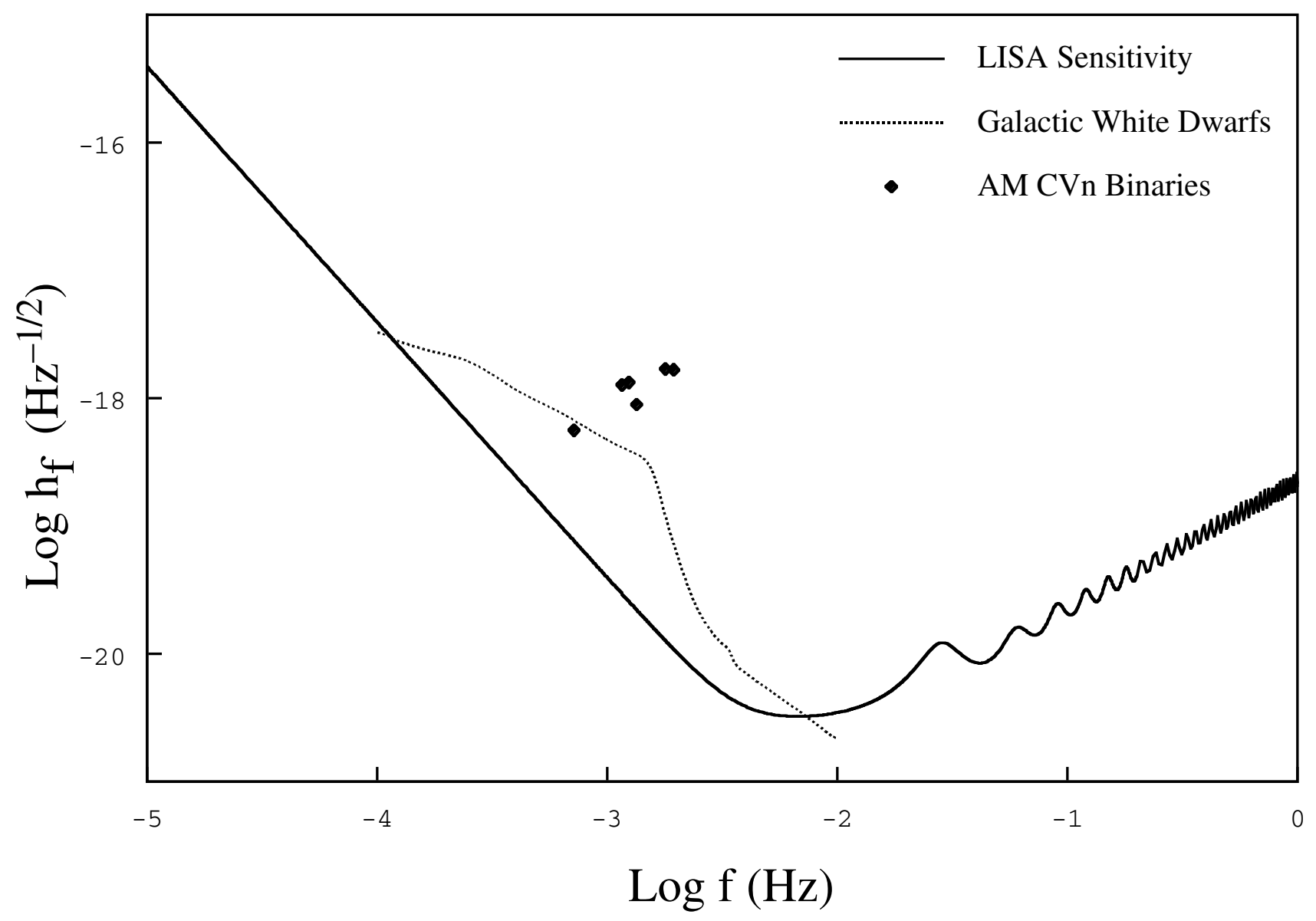




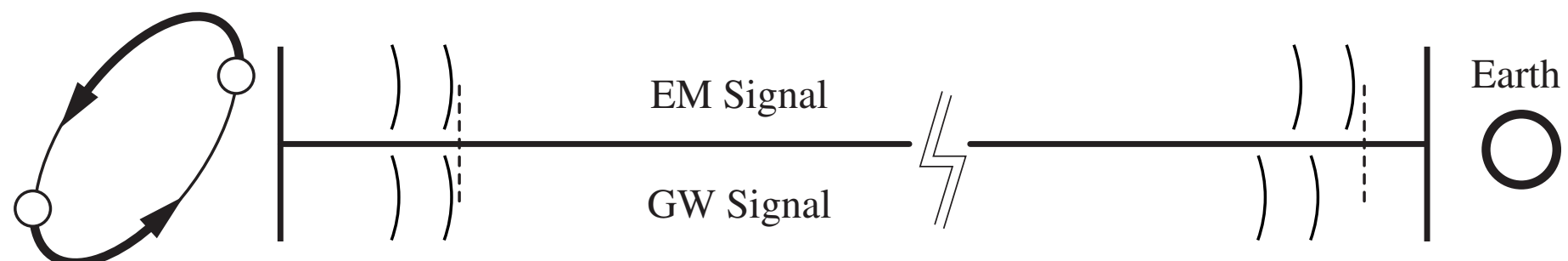

Binary 


$$
0
$$




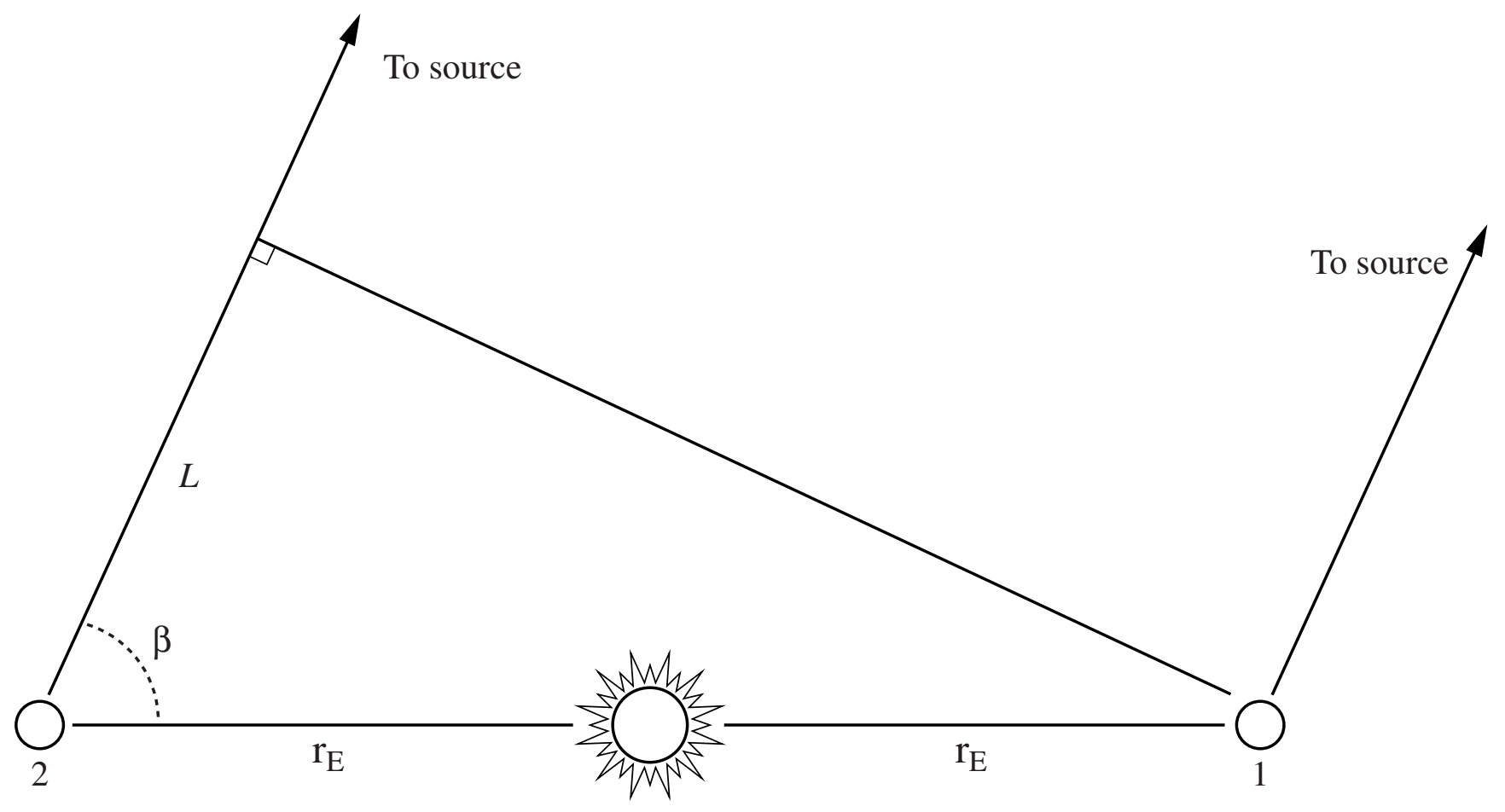

